\title{
Asymptotic Bohr Radius for the Polynomials in One Complex Variable
}

\author{
Cheng Chu
}

\begin{abstract}
We consider the Bohr radius $R_{n}$ for the class of complex polynomials in one variable of degree at most $n$. It was conjectured by R. Fournier in 2008 that $R_{n}=\frac{1}{3}+\frac{\pi^{2}}{3 n^{2}}+o\left(\frac{1}{n^{2}}\right)$. We shall prove this conjecture is true in this paper.
\end{abstract}

\section{Introduction}

Let $\mathbb{D}$ be the open unit disk in the complex plane $\mathbb{C}$ and $H^{\infty}$ be the Banach space of bounded analytic functions on $\mathbb{D}$ with the norm

$$
\|f\|_{\infty}=\sup _{z \in \mathbb{D}}|f(z)| .
$$

Also let $\mathcal{P}_{n}$ denote the subspace of $H^{\infty}$ consisting of all the complex polynomials of degree at most $n$. The Bohr radius $R$ for $H^{\infty}$ is defined as

$$
R=\sup \left\{r \in(0,1): \sum_{k=0}^{\infty}\left|a_{k}\right| r^{k} \leqslant\|f\|_{\infty}, \text { for all } f(z)=\sum_{k=0}^{\infty} a_{k} z^{k} \in H^{\infty}\right\} .
$$

Bohr's famous power series theorem [1] shows that $R=\frac{1}{3}$.

In 2004, Guadarrama [4] considered the Bohr type radius for the class $\mathcal{P}_{n}$ defined by

$$
R_{n}=\sup \left\{r \in(0,1): \sum_{k=0}^{n}\left|a_{k}\right| r^{k} \leqslant\|p\|_{\infty}, \text { for all } p(z)=\sum_{k=0}^{n} a_{k} z^{k} \in \mathcal{P}_{n}\right\},
$$

and gave the estimate

$$
\frac{C_{1}}{3^{n / 2}}<R_{n}-\frac{1}{3}<C_{2} \frac{\log n}{n},
$$

for some positive constants $C_{1}$ and $C_{2}$. Later in 2008, Fournier obtained an explicit formula for $R_{n}$ by using the notion of bounded preserving operators. He proved the following theorem $[\mathbf{2}]$

2010 Mathematics Subject Classification. Primary 41.

Partially supported by National Science Foundation Grant DMS 1300280. 
Theorem 1.1. For each $n \geqslant 1$, let $T_{n}(r)$ be the following $(n+1) \times(n+1)$ symmetric Toeplitz matrix

$$
\left(\begin{array}{cccccc}
1 & r & -r^{2} & r^{3} & \cdots & (-1)^{n-1} r^{n} \\
r & 1 & r & -r^{2} & \cdots & (-1)^{n-2} r^{n-1} \\
-r^{2} & r & 1 & r & & \vdots \\
r^{3} & -r^{2} & r & 1 & \ddots & \vdots \\
\vdots & & \ddots & \ddots & \ddots & \\
(-1)^{n-1} r^{n} & & \cdots & & r & 1
\end{array}\right)
$$

Then $R_{n}$ is equal to the smallest root in $(0,1)$ of the equation

$$
\operatorname{det} T_{n}(r)=0 \text {. }
$$

Based on the numerical evidence, he conjectured that

$$
R_{n}=\frac{1}{3}+\frac{\pi^{2}}{3 n^{2}}+\ldots
$$

The purpose of this note is to provide a positive answer. We shall prove

Theorem 1.2. Let $R_{n}$ be as in (1.1), then

$$
\lim _{n \rightarrow \infty} n^{2}\left(R_{n}-\frac{1}{3}\right)=\frac{\pi^{2}}{3}
$$

\section{Main Theorem}

In this section, we prove Theorem 1.2. The methods we use are similar to that in $[3$, Chapter 5].

Proof of Theorem 1.2. Let $\Delta_{n}=\Delta_{n}(r)=\operatorname{det} T_{n}(r)$, where $T_{n}(r)$ is the symmetric Toeplitz matrix (1.2). By Theorem 1.1, $R_{n}$ is the smallest root in $(0,1)$ of the equation

$$
\Delta_{n}(r)=0 .
$$

For $n \geqslant 2$, multiplying the second row of $\Delta_{n}$ by $\mathrm{r}$, adding it to the first row and performing a similar operation with the columns, we have

$$
\begin{gathered}
\Delta_{n}(r)=\operatorname{det}\left(\begin{array}{cccccc}
1+3 r^{2} & 2 r & 0 & & \cdots & 0 \\
2 r & 1 & r & -r^{2} & \cdots & (-1)^{n-2} r^{n-1} \\
0 & r & 1 & r & & \\
& -r^{2} & r & 1 & \ddots & \vdots \\
\vdots & & \ddots & \ddots & \ddots & \\
0 & & \cdots & & r & 1
\end{array}\right) \\
=\left(3 r^{2}+1\right) \Delta_{n-1}(r)-4 r^{2} \Delta_{n-2}(r) .
\end{gathered}
$$

If we set $\Delta_{-1}(r)=1$, then the recurrence relation (2.2) holds for all $n \geqslant 1$. 
Consider the function associated with these Toeplitz matrices $\Delta_{n}$

$$
f(r, \theta)=1+\sum_{|n|>0}(-1)^{n-1} r^{n} e^{i n \theta}=\frac{3 r^{2}+4 r \cos \theta+1}{r^{2}+2 r \cos \theta+1} .
$$

In order to solve the equation (2.1), suppose

$$
r=g(x)=\frac{1}{3}\left(-2 \cos x-\sqrt{4 \cos ^{2} x-3}\right),
$$

for some $x \in[0, \pi]$. This substitution comes from

$$
3 r^{2}+4 r \cos x+1=0=f(r, x) .
$$

(In fact, for a fixed $r$, every eigenvalue $\lambda$ of $T_{n}$ can be written as $\lambda=f(r, x)$, for some $x \in[0, \pi]$. See $[\mathbf{3}$, Chapter 5].)

Then (2.2) becomes

$$
\Delta_{n}=(-4 r \cos x) \Delta_{n-1}-4 r^{2} \Delta_{n-2} .
$$

Its characteristic equation

$$
\lambda^{2}+4 r \cos x \lambda+4 r^{2}=0
$$

has the roots $-2 r e^{ \pm i x}$. Adding the initial conditions $\Delta_{-1}=\Delta_{0}=1$, we have

$$
\Delta_{n}=\frac{(-2 r)^{n+1}}{1-r^{2}}\left(\frac{\sin (n+2) x}{\sin x}+2 r \frac{\sin (n+1) x}{\sin x}+r^{2} \frac{\sin n x}{\sin x}\right) \text {. }
$$

Denote

$$
p_{n}(\cos x)=\frac{\sin (n+2) x}{\sin x}+2 r \frac{\sin (n+1) x}{\sin x}+r^{2} \frac{\sin n x}{\sin x} .
$$

Then $p_{n}(t)$ is a polynomial of degree $n+1$ in $t=\cos x$. Let

$$
x_{\nu}=\frac{\nu \pi}{n+2}, \quad \nu=1,2, \cdots, n+1 .
$$

Direct computation shows that

$$
p_{n}\left(\cos x_{\nu}\right)=(-1)^{\nu} 2 r(1+r \cos \nu)
$$

thus

Also

$$
\operatorname{sgn} p_{n}\left(\cos x_{\nu}\right)=(-1)^{\nu}
$$

$$
\lim _{x \rightarrow 0^{+}} p_{n}(\cos x)>0 .
$$

So $p_{n}$ has $n+1$ distinct zeros $\left\{\cos t_{\nu}^{(n)} \mid \nu=1,2, \cdots, n+1\right\}$, such that

$$
0<t_{1}^{(n)}<x_{1}<t_{2}^{(n)}<x_{2}<\cdots<t_{n+1}^{(n)}<x_{n+1}<\pi .
$$

That means every root of the equation (2.1) has the form (2.3). Notice that $g$ is positive only on $\left[\frac{5 \pi}{6}, \pi\right]$ and decreasing on $\left[\frac{5 \pi}{6}, \pi\right]$, so the smallest root of $(2.1)$ in the interval $(0,1)$ is $g\left(t_{n+1}^{(n)}\right)$, i.e. $R_{n}=g\left(t_{n+1}^{(n)}\right)$.

Next, we will find an asymptotic expression for $t_{n+1}^{(n)}$. Notice that

$$
\lim _{n \rightarrow \infty}(-1)^{n+1} \frac{p_{n}\left(-\cos \frac{z}{n+2}\right)}{n+2}=(1-r)^{2} \frac{\sin z}{z} .
$$


And (2.5) holds uniformly for $|z|<2 \pi$. Let

$$
t_{n+1}^{(n)}=\frac{(n+1) \pi-\epsilon_{n}}{n+2},
$$

then $\epsilon_{n} \in(0, \pi)$ by relation (2.4). Thus

$$
\begin{aligned}
0 & =\lim _{n \rightarrow \infty}(-1)^{n+1} \frac{p_{n}\left(\cos \frac{(n+1) \pi-\epsilon_{n}}{n+2}\right)}{n+2} \\
& =\lim _{n \rightarrow \infty}(-1)^{n+1} \frac{p_{n}\left(-\cos \frac{\pi+\epsilon_{n}}{n+2}\right)}{n+2} \\
& =\lim _{n \rightarrow \infty}(1-r)^{2} \frac{\sin \left(\pi+\epsilon_{n}\right)}{\pi+\epsilon_{n}} .
\end{aligned}
$$

Hence the accumulation point of $\left\{\epsilon_{n}\right\}$ is either 0 or $\pi$. Let

$$
y_{n}=\frac{(n+1) \pi-\frac{\pi}{2}}{n+2}, \quad y_{n} \in\left(x_{n}, x_{n+1}\right) .
$$

Using (2.5) again, we have

$$
\begin{aligned}
& \lim _{n \rightarrow \infty}(-1)^{n+1} \frac{p_{n}\left(\cos \frac{(n+1) \pi-\frac{\pi}{2}}{n+2}\right)}{n+2} \\
= & \lim _{n \rightarrow \infty}(-1)^{n+1} \frac{p_{n}\left(-\cos \frac{\frac{3 \pi}{2}}{n+2}\right)}{n+2} \\
= & \lim _{n \rightarrow \infty}(1-r)^{2} \frac{\sin \left(\frac{3 \pi}{2}\right)}{\frac{3 \pi}{2}}<0 .
\end{aligned}
$$

When $n$ is sufficiently large,

$$
\operatorname{sgn} p_{n}\left(\cos y_{n}\right)=(-1)^{n}=\operatorname{sgn} p_{n}\left(\cos x_{n}\right),
$$

so $t_{n+1}^{(n)} \in\left(y_{n}, x_{n+1}\right)$. Consequently, $\epsilon_{n} \rightarrow 0$ as $n \rightarrow \infty$, and then

$$
R_{n}=g\left(\pi-\theta_{n}\right)
$$

where

$$
\theta_{n}=\frac{\pi+\epsilon_{n}}{n+2}=\frac{\pi}{n}+o\left(\frac{1}{n}\right), \quad \text { as } n \rightarrow \infty .
$$

By $(2.3)$,

$$
\begin{aligned}
R_{n} & =\frac{1}{3}\left(2 \cos \theta_{n}-\sqrt{4 \cos ^{2} \theta_{n}-3}\right) \\
& =\frac{1}{3}+\frac{\pi^{2}}{3 n^{2}}+o\left(\frac{1}{n^{2}}\right) .
\end{aligned}
$$




\section{References}

[1] H. Bohr, A theorem concerning power series, Proc. Lond. Math. Soc. (2) 13 (1914), 1-5.

[2] R. Fournier, Asymptotics of the Bohr radius for polynomials of fixed degree, J. Math. Anal. Appl. 338 (2008), 1100-1107.

[3] U. Grenander and G. Szegö, Toeplitz forms and their applications, University of California Press, Berkeley, 1958.

[4] Z. Guadarrama, Bohr's radius for polynomials in one complex variable, Comput. Methods Funct. Theory 5 (2007), 143-151.

Department of Mathematics, Washington University in Saint Louis, Saint Louis, Missouri, USA

E-mail address: chengchu@math.wustl.edu 